\title{
Titres de spécialiste pour tous?
}

\author{
Octroi de titres postgrades fédéraux à des médecins sans titre en vertu des dispositions transitoires
}

Ch. Hänggeli, responsable du secrétariat pour la formation prégraduée, postgraduée et continue (FPPC)

* Dans leur étude, le Dr Ingrid Laura Wyler-Brem et al. ont constaté que les non-porteurs de titre interrogés avaient accompli une formation postgraduée moyenne de 5,2 ans.

\section{Références}

1 Loi fédérale du 19 décembre 1877 concernant l'exercice des professions de médecin, de pharmacien et de vétérinaire dans la Confédération suisse.

2 Ordonnance sur la formation postgrade et la reconnaissance des diplômes et des titres postgrades des professions médicales.

3 Wyler-Brem IL, et al. Äquivalenzausweis für ÄrztInnen ohne FMH-Titel. Ars Medici 1998;16:969.

4 Hänggeli C. Quels critères pour l'examen de spécialiste? Bull Méd Suisses 2002; 83(5):189-90.
L'entrée en vigueur simultanée au $1^{\text {er }}$ juin 2002 des accords bilatéraux et de la loi révisée sur les professions médicales (LEPM) [1] avec son ordonnance [2] institue de nouvelles bases pour l'admission des médecins à pratiquer à titre indépendant. En effet, pendant plus de 120 ans, il suffisait d'être détenteur d'un diplôme fédéral de médecin pour obtenir le droit d'ouvrir un cabinet médical. Ces temps sont désormais révolus car pour exercer une activité indépendante il faudra désormais être porteur d'un titre postgrade fédéral ou d'un titre de formation postgraduée étranger reconnu (cf. tab. 1). En outre, les cantons n'auront plus la compétence d'attribuer à titre exceptionnel des autorisations de pratiquer à des médecins non-porteurs d'un titre fédéral ou d'un diplôme de médecin reconnu.

Un changement si radical sur le plan légal exige des dispositions transitoires très détaillées, tenant compte des réalités. Avec l'article 24 de la LEPM, le législateur a fait preuve de souplesse en reconnaissant, d'une part, les titres de spécialiste FMH en tant que titres postgrades fédéraux et, d'autre part, en proposant une solution généreuse pour tous les médecins en pratique privée sans titre de spécialiste.

\section{Les titres de spécialiste FMH n'ont-ils plus aucune valeur?}

Les titres FMH de droit privé ont fait leur temps et ne sont plus attribués depuis le $1^{\text {er }}$ juin 2002 . Ils ont été remplacés par 44 titres postgrades fédéraux (voir tableau). Les conditions nécessaires à leur attribution demeurent identiques à deux exceptions près: désormais, les futurs spécialistes ne doivent plus rédiger de thèse de doctorat et l'affiliation à la FMH est facultative. Les méde- cins déjà porteurs d'un titre de spécialiste FMH sont reconnus d'office. Il n'est pas nécessaire d'acquérir un nouveau diplôme, étant donné que les titres de spécialiste FMH - et seulement ceux-ci - sont équivalents aux titres postgrades fédéraux.

\section{Quelles sont les conséquences pour les médecins praticiens sans titre de spécialiste?}

A l'heure actuelle, on compte en Suisse environ 2000 médecins qui, pour diverses raisons, exercent en pratique privée* [3] sans être porteurs d'un titre de spécialiste FMH, d'ailleurs non exigé par la loi jusqu'ici. L'article 24 de la LEPM garantit à ces médecins de pouvoir continuer à pratiquer sans titre de spécialiste, mais leur offre aussi la possibilité de demander un titre postgrade fédéral correspondant à leur «formation postgraduée pratique et théorique». Le législateur entend ainsi éviter les éventuelles discriminations dont pourraient faire l'objet les médecins sans titre en raison de l'entrée en vigueur des nouveaux titres postgrades fédéraux et de la libre circulation des personnes au sein de l'Union européenne.

A l'article 11 de l'ordonnance à la LEPM, le Conseil fédéral a édicté des dispositions détaillées mentionnant les personnes qui pourront obtenir un titre postgrade fédéral selon les dispositions transitoires (cf. texte en annexe), et à quelles conditions (facilitées).

Grâce à cette solution, on peut supposer que tous les médecins praticiens de Suisse disposeront d'un titre fédéral postgrade dans quelques années et pourront figurer dans le registre des détenteurs d'un titre postgrade fédéral. 


\section{En tant que médecin praticien sans titre de spécialiste, comment puis-je obtenir un titre postgrade fédéral?}

\section{Ordonnance sur la formation postgrade et la reconnaissance des diplômes et des titres postgrades des professions médicales}

Art. 11 Octroi des titres postgrades fédéraux en vertu des dispositions transitoires

1 Quiconque exerçait la profession de médecin à titre indépendant en Suisse avant le $1^{\text {er }}$ juin 2002, peut, pour autant qu'il n'ait pas obtenu de titre postgrade visé à l'art. 9 avant cette date, demander un titre fédéral.

2 Les personnes habilitées à présenter une demande se voient octroyer le titre de «médecin praticien» si elles ne reçoivent pas un titre visé aux al. 4 à 6 .

3 Peuvent être validés comme formation postgrade exigée pour un titre visé à l'art. 1 let. a:

a. jusqu'à une année d'activité pratique exercée de manière indépendante;

b. opérations, examens, etc., exécutés de manière indépendante, à raison d'un tiers.

Pour que le titre puisse être octroyé, les autres conditions en matière de formation postgrade fixées dans le programme applicable doivent être remplies.

4 Quiconque a accompli au moins deux années de formation postgrade pouvant être validées pour le titre de spécialiste en médecine générale et, par année de formation postgrade manquante, pratiqué pendant deux ans de manière indépendante et prépondérante dans les soins de base, se voit octroyer sans autres formalités le titre de médecin spécialiste en «médecine générale».

5 Quiconque a accompli au moins trois années de formation postgrade pouvant être validées pour le titre de spécialiste en psychiatrie et psychothérapie d'enfants et d'adolescents et qui, pour chaque année manquante de formation postgrade, a pratiqué de manière indépendante et prépondérante pendant deux années dans le domaine en question, se voit octroyer sans autres formalités le titre postgrade correspondant, s'il peut, en plus, justifier de 150 heures de supervision et d'une expérience psychothérapeutique personnelle.

6 Quiconque ne remplit pas les conditions visées aux al. 3 à 5 mais a pratiqué de manière indépendante et prédominante dans le domaine en question pendant au moins cinq années peut obtenir un titre de spécialiste fédéral en passant l'examen correspondant.

7 Les conditions relatives à l'obtention d'un titre postgrade visé aux al. 2 à 6 doivent être remplies le 31 décembre 2007 au plus tard. A partir de 2002, il est exigé en sus 80 heures par année de formation postgrade conformément aux exigences de l'organisme responsable de la formation postgrade.

Vu la situation particulière des 2000 médecins praticiens sans titre de spécialiste, la formulation de l'article 11 de l'ordonnance à la loi sur l'exercice des professions médicales a été établie de manière différenciée et peut sembler quelque peu compliquée.

Par souci de clarté, nous exposons ci-après, en premier lieu, les conditions nécessaires à l'obtention d'un titre selon l'article $11,1^{\mathrm{er}}$ al., de l'ordonnance à la LEPM (chiffre 1). Vous ne pourrez être mis au bénéfice des dispositions transitoires que si vous remplissez les conditions exigées pour l'obtention facilitée d'un titre. Le titre qui pourra vous être octroyé jusqu'à la fin 2007 au plus tard dépendra finalement des possibilités énumérées aux points 2 à 5 . 


\section{Conditions de base et attribution du titre postgrade fédéral de «médecin praticien»}

\section{(article 11, al. 1 et 2 , de l'ordonnance)}

Vous pouvez demander un titre postgrade fédéral à des conditions facilitées, si vous exerciez déjà la profession de médecin à titre indépendant avant le $1^{\text {er }}$ juin 2002 et ne disposez pas d'un titre de spécialiste (art. 11, $1^{\mathrm{er}}$ al., LEPM).

Que signifie «exercer la profession de médecin à titre indépendant»?

- En tant que détenteur d'une autorisation cantonale de pratiquer, vous exercez en Suisse une activité médicale à votre propre nom et sous votre propre responsabilité. Pour avoir le droit de pratiquer, une autorisation cantonale de pratiquer (sans limitations de temps, de lieu ou de compétences!) et un numéro de code créancier suffisent. Si vous ne pratiquez pas à la charge de l'assurance de base et, par conséquent, ne disposez pas d'un numéro de code créancier, vous devez demander une attestation à votre société cantonale de médecine ou à la direction des affaires sanitaires de votre canton.

- Si vous n'exercez pas en votre propre nom et sous votre propre responsabilité, mais êtes salarié conformément à la loi sur le travail, votre activité peut exceptionnellement être considérée comme «indépendante» si elle correspond effectivement à celle en pratique privée. La condition étant un contrat de travail pour une durée indéterminée en tant que médecin dirigeant (pas chef de clinique) et la pratique d'une activité indépendante. Vous êtes donc appelé, par exemple en tant que responsable d'une HMO ou d'une clinique de jour, à prendre des décisions d'ordre médical sous votre propre responsabilité. Les attestations appropriées de l'employeur et de la direction des affaires sanitaires doivent être présentées.

Que signifie «pour autant qu'il n'ait pas obtenu de titre postgrade avant cette date»?

Le médecin qui dispose déjà d'un titre de formation postgraduée, mais qui exerce dans une autre spécialité peut renoncer à son ancien titre et demander, en vertu des dispositions transitoires, un titre correspondant à son domaine actuel d'activité. La présentation des attestations appropriées demeure réservée.
Si vous remplissez les deux conditions précitées, vous avez droit au titre fédéral de «médecin praticien». Ce titre n'a d'intérêt que dans la mesure où vous ne remplissez pas (encore) les exigences pour l'obtention facilitée d'un titre de spécialiste conformément aux points 2 à 4 ci-après.

\section{Comment obtenir le titre postgrade fédéral en «médecine générale»?}

\section{(article 11, $4^{\mathrm{e}}$ al., de l'ordonnance)}

Si vous avez accompli au moins deux ans de formation postgraduée selon le programme de formation en médecine générale et que, par année de formation manquante, vous avez pratiqué pendant deux ans de manière indépendante et prépondérante dans les soins de base, vous pouvez obtenir sans autres formalités le titre de spécialiste en médecine générale.

Que signifie «formation postgrade pouvant être validée»?

Toute formation postgraduée attestée et reconnue depuis la création du titre de spécialiste en médecine générale peut être validée, sous réserve de la présentation de certificats correspondants (du moins sous la forme acceptée jusqu'à présent). Sans la présentation desdites attestations, la validation est exclue.

Que signifie «de manière indépendante et prépondérante dans les soins de base»? Vous devez fournir une déclaration écrite mentionnant que vous avez accompli cette activité de manière indépendante et prépondérante et pour la durée exigée dans le domaine de la médecine de premier recours. En cas d'activité à temps partiel, la durée exigée est rallongée en conséquence. (1600 h de travail annuel correspondent à une activité à plein temps. N.B. le TARMED est établi sur la base d'un temps de travail total de 1976 heures.)

\section{Comment obtenir le titre de spécia- liste en «psychiatrie et psychothérapie ou en psychiatrie et psychothérapie d'enfants et d'adolescents»?}

(article 11, $5^{\mathrm{e}}$ al., de l'ordonnance)

Si vous avez accompli au moins trois ans de formation postgraduée conformément au programme de formation en psychiatrie et psychothérapie (ou en psychiatrie et psychothérapie 
d'enfants et d'adolescents) et que, pour chaque année de formation manquante, vous avez pratiqué de manière indépendante et prépondérante pendant 2 ans dans le domaine en question et que vous justifiez, en plus, de 150 heures de supervision et d'une expérience psychothérapeutique propre, vous obtiendrez sans autres formalités le titre postgrade correspondant.

Les formulations «formation postgrade pouvant être validée» et «de manière indépendante et prépondérante dans le domaine en question» sont expliquées au point 2 .

\section{A quelles conditions peut-on obtenir les autres titres fédéraux de spécialistes?}

\section{(article 11, al. 3 et 6, de l'ordonnance)}

Les autres titres fédéraux de spécialiste peuvent être octroyés aux médecins sans titre à des conditions facilitées. Autrement dit:

\section{Variante 1}

Vous devez répondre à toutes les exigences du programme de formation postgraduée en vigueur et votre formation sera évaluée au même titre que celle des candidats «ordinaires» avec néanmoins deux allégements:

- le «remplacement» de n'importe quelle année de formation postgraduée (manquante) par une année d'activité pratique;

- la validation, à raison d'un tiers, des opérations, examens, etc., exécutés de manière indépendante.

Tant l'activité pratique indépendante que les opérations et les examens effectués de manière indépendante doivent être attestés ou faire l'objet d'une déclaration écrite de votre part.

\section{Variante 2}

Si vous avez pratiqué de manière indépendante et prépondérante dans le domaine concerné pendant au moins cinq ans, vous pouvez obtenir un titre fédéral de spécialiste en passant l'examen en question. Veuillez en outre tenir compte que, pour certains examens, seuls sont admis les candidats répondant en plus à certaines exigences spécifiques (p. ex. liste complète des opérations en gynécologie et obstétrique).

Est valable uniquement le certificat délivré par la société de discipline médicale compétente attestant la réussite de l'examen de spécialiste. Il doit notamment s'agir d'un examen dont le caractère éliminatoire est déjà entré en vigueur [4].

La formulation «pratiquer de manière indépendante et prédominante dans le domaine en question» est expliquée au point 2 .
Tableau 1

Les 44 titres postgrades fédéraux.

\begin{tabular}{|c|c|}
\hline Allergologie et immunologie clinique & 6 ans \\
\hline Anesthésiologie & 6 ans \\
\hline Angiologie & 6 ans \\
\hline Cardiologie & 6 ans \\
\hline Chirurgie cardiaque et vasculaire thoracique & 6 ans \\
\hline Chirurgie maxillo-faciale & 6 ans \\
\hline Chirurgie pédiatrique & 6 ans \\
\hline $\begin{array}{l}\text { Chirurgie plastique, reconstructive } \\
\text { et esthétique }\end{array}$ & 6 ans \\
\hline Chirurgie & 6 ans \\
\hline Chirurgie orthopédique & 6 ans \\
\hline Dermatologie et vénéréologie & 5 ans \\
\hline Endocrinologie-diabétologie & 6 ans \\
\hline Gastroentérologie & 6 ans \\
\hline Génétique médicale & 5 ans \\
\hline Gynécologie et obstétrique & 6 ans \\
\hline Hématologie & 6 ans \\
\hline Infectiologie & 6 ans \\
\hline Médecin praticien & 2 ans \\
\hline Médecine du travail & 5 ans \\
\hline Médecine générale & 5 ans \\
\hline Médecine intensive & 6 ans \\
\hline Médecine interne & 5 ans \\
\hline Médecine légale & 5 ans \\
\hline Médecine nucléaire & 5 ans \\
\hline Médecine pharmaceutique & 5 ans \\
\hline Médecine physique et de réadaptation & 5 ans \\
\hline Médecine tropicale & 5 ans \\
\hline Néphrologie & 6 ans \\
\hline Neurochirurgie & 6 ans \\
\hline Neurologie & 6 ans \\
\hline Oncologie médicale & 6 ans \\
\hline Ophtalmologie & 5 ans \\
\hline Oto-rhino-laryngologie & 5 ans \\
\hline Pathologie & 6 ans \\
\hline Pédiatrie & 5 ans \\
\hline Pharmacologie et toxicologie cliniques & 6 ans \\
\hline Pneumologie & 6 ans \\
\hline Prévention et santé publique & 5 ans \\
\hline $\begin{array}{l}\text { Psychiatrie et psychothérapie d'enfants } \\
\text { et d'adolescents }\end{array}$ & 6 ans \\
\hline Psychiatrie et psychothérapie & 6 ans \\
\hline Radiologie & 6 ans \\
\hline Radio-oncologie / radiothérapie & 6 ans \\
\hline Rhumatologie & 6 ans \\
\hline Urologie & 6 ans \\
\hline
\end{tabular}




\section{Formation continue: une exigence supplémentaire}

(article 11, $7^{\mathrm{e}}$ al., de l'ordonnance)

Si vous soumettez votre demande de spécialiste après le 31 décembre 2002 (ou si vous n'avez pas entièrement rempli les conditions exigées jusqu'à cette date), vous devez attester, en plus des exigences fixées pour l'année 2002, 80 heures par année de formation continue conformément aux dispositions de la Réglementation pour la formation continue.

Exemple: si vous présentez une demande de titre en 2003 (ou ne remplissez les conditions exigées qu'à ce moment-là), il vous faudra demander à la société de discipline médicale compétente une attestation de la formation continue accomplie en 2002. Pour les demandes déposées en 2007 (dernier délai), l'attestation de formation continue devra comprendre les années 2002 à 2006.

\section{Dispositions transitoires}

(article 11, $7^{\mathrm{e}}$ al., de l'ordonnance)

Veuillez en outre tenir compte du fait que les conditions exigées pour l'obtention d'un titre postgrade fédéral doivent être remplies jusqu'au 31 décembre 2007 au plus tard.

Si lesdites conditions ne sont pas remplies jusqu'à la date précitée, l'octroi du titre ne se fera plus qu'en vertu des dispositions ordinaires.

\section{A qui dois-je envoyer ma demande de titre?}

En raison du nombre important de demandes de titre, nous vous prions de bien vouloir présenter votre demande de titre exclusivement au moyen du formulaire figurant sur le site internet de la FMH (www.fmh.ch/awf). Nous ne pourrons garantir un traitement rapide de votre demande que si vous fournissez l'ensemble des documents et des informations exigés dans le formulaire précité.

Approuvé par le Comité central de la FMH le 24 avril 2002. 